\title{
Systèmes de régulation et solidarité pour les filières laitières biologiques
}

Some examples and thinking about the system of control and solidarity in the organic milk farming sector

\section{Claire Touret}

\section{(2) OpenEdition}

\section{Journals}

Édition électronique

URL : https://journals.openedition.org/economierurale/4225

DOI : 10.4000/economierurale.4225

ISSN : 2105-2581

Éditeur

Société Française d'Économie Rurale (SFER)

Édition imprimée

Date de publication : 20 janvier 2014

Pagination : 49-63

ISSN : 0013-0559

Référence électronique

Claire Touret, "Systèmes de régulation et solidarité pour les filières laitières biologiques », Économie rurale [En ligne], 339-340 | janvier-mars 2014, mis en ligne le 20 janvier 2016, consulté le 21 septembre 2021. URL : http://journals.openedition.org/economierurale/4225 ; DOI : https://doi.org/10.4000/ economierurale.4225

(c) Tous droits réservés 


\title{
Systèmes de régulation et solidarité pour les filières laitières biologiques
}

\author{
Claire TOURET • Fédération nationale d'agriculture biologique (FNAB), Paris \\ ctouret@fnab.org
}

Dans les années 2001-2007, les prix de nombreuses productions biologiques ont fortement baissé et certains producteurs n'avaient plus de valorisation en bio. Des initiatives ont vu le jour avec l'objectif de sécuriser les producteurs par rapport aux surcoûts spécifiques liés à ce mode de production, de maintenir un volume de produits disponibles pour de nouveaux marchés et d'assurer solidarité et cohérence territoriale pour le maintien d'une dynamique de développement de l'agriculture biologique. À partir de l'exemple de la Caisse de solidarité des éleveurs laitiers bio lorrains mise en place en 2003, de l'expérience de Biolait et des dynamiques en cours, la Fédération nationale de l'agriculture biologique propose une réflexion sur l'organisation des éleveurs laitiers bio qui s'inscrit dans un contexte de mutations globales des filières agricoles et une réflexion sur la valorisation des performances plurielles de la bio croisant dynamique nationale et projet de territoire.

MOTS-CLÉS : solidarité, filière, éleveurs laitiers, biologique, nouvelle régulation

\section{Some examples and thinking about the system of control and solidarity in the orga- nic milk farming sector}

Between 2001 and 2007 the price of many organic products has gone down so much that a few producers thought it wasn't worth the trouble growing such products any longer. Some initiatives have been developed since with the following aims: secure the producers a minimum about the incremental cost related to an organic production, maintain a volume of products available to gain new markets, ensure solidarity and territorial cohesion in order to keep a dynamic development in organic farming. Starting from the example of the Lorraine pooling case for dairy farmers, the Biolait experience and the current dynamics, the FNAB proposes to think about the dairy farmer organization which happens in a context of global change in the agricultural sectors. This reflection is also within the FNAB in a more comprehensive way on the development of plural systemic performance of Bio farming as both national and territorial projects. (JEL: Q13).

KEYWORDS: solidarity, organic, milk sector, farmer organization, new regulation

$E^{n}$ 2011, à l'occasion du colloque de clôture de l'Assemblée générale de la Fédération nationale d'agriculture biologique (FNAB) des régions de France ${ }^{1}$, à Amiens, Philippe Lacombe, professeur

1. Depuis 1978, la Fédération Nationale d'Agriculture Biologique des régions de France (FNAB) fédère les agrobiologistes, toutes filières de production confondues, via les organisations régionales adhérentes qui la composent. La FNAB promeut et défend le métier d'agrobiologiste. En tant que structure de développement, elle met en place des actions et apporte son expertise afin de développer l'agriculture biologique partout et pour tous, de façon cohérente et durable. d'économie, ancien directeur scientifique de l'INRA et président du groupe prospective DATAR « agriculture et territoire », a interpellé le mouvement bio sur sa capacité de changement. Il a indiqué les étapes que l'agriculture biologique avait passées avec succès : émergence des techniques et pratiques de l'agriculture bio, reconnaissance par le public et prise en considération par les politiques. Pour passer du prototype au modèle, il a rappelé combien il était nécessaire de changer d'échelle.

En sociologie (Buck et al., 1997 ; Raynolds, Laura, 2009) la notion d'échelle 
est utilisée pour décrire les mutations que connaissent les initiatives en phase d'expansion comme le commerce équitable et l'agriculture biologique. Cette croissance implique de travailler avec de nouveaux acteurs et peut créer des tensions de diverses natures. L'agriculture biologique traverse aujourd'hui cette évolution, mais ce n'est pas la première fois. Considérer les mécanismes liés aux changements d'échelle pour les gérer permet de les accompagner, en évitant que les pratiques de l'alternative de départ ne se dégradent. C'est en tout cas le pari de la FNAB. Plusieurs mécanismes sont en jeu. Le Velly (2009) en cite deux particulièrement : la certification et la délégation, notamment commerciale. Nous nous intéressons dans cet article à ce deuxième mécanisme. Comment les producteurs bio s'organisent pour la mise en marché de leur production ? À qui, quoi et comment délèguent-ils cette tâche ? Nous limiterons l'examen de l'organisation des producteurs bio au secteur du lait de vache. Celui-ci est particulièrement touché par le démantèlement des outils européens de gestion des marchés et doit affronter une mutation importante. Cette orientation, lourde de remise en cause progressive des dispositifs actuels de régulation du secteur agricole, légitime également l'interrogation sur de nouveaux modèles d'organisation des filières, combinant une régulation privée et une nouvelle articulation avec les politiques publiques sur les territoires. Pour les éleveurs bio, le défi est donc double.

Le changement d'échelle de la bio, selon la FNAB, ne peut pas être pensé par la seule logique de compétitivité du prix. Cette logique aboutit, à force de pressions et de tensions, à des systèmes de production qui négligent les aspects environnementaux et sociaux. Elle conduit aussi à nier le rôle des fermes au sein des territoires, en termes de construction de bien public à long terme (qualité des sols, de l'air et de l'eau) et d'identité culturelle (paysages, réseaux sociaux, liens producteurs consommateurs...). Pour qu'une économie agroalimentaire soit durable, il faut qu'elle permette une valorisation des performances environnementales et sociales. Milanesi (2012), économiste et maître de conférences à l'université Paul Sabatier (Toulouse), a exploré le concept d'externalité. Il s'est posé la question de sa pertinence pour construire une rémunération pérenne des performances plurielles de la bio. L'externalité permet surtout de compenser, à la marge, les effets positifs ou négatifs, mais néglige ou marginalise les interdépendances entre activité économique, territoire, milieu social et environnement. Or c'est l'inverse qui est vrai. L'activité agricole est interdépendante des milieux sociaux et environnementaux. Les agriculteurs biologiques vivent et gèrent ceux-ci dans leurs pratiques. Ils ont l'ambition de valoriser ces performances plurielles. Le concept d'externalité positive ne le permet pas, vu qu'il les considère à la marge. Fruit de la rencontre entre des économistes et des écologues, le concept d'interdépendance amène à penser que les ressources, telles que l'eau, la fertilité des sols, sont communes aux usagers du territoire et qu'elles engagent une responsabilité collective. Il nous invite donc à construire de nouvelles organisations entre acteurs (au sens large) capables de gérer des ressources communes au niveau des territoires et des filières de production. Il guidera notre analyse de l'organisation économique de producteurs bio : comment cherchent-ils à valoriser leurs performances plurielles, à co-responsabiliser leurs partenaires, à gérer les complémentarités entre des échelles territoriales différentes?

Dans un premier temps, nous exposerons le contexte du secteur laitier en plein bouleversement et nous décrirons les spécificités du secteur laitier biologique. Nous analyserons, dans un deuxième temps, les premières expériences d'organisations économiques, collectives, d'éleveurs laitiers 
bio. Puis, nous développerons les structurations plus récentes de ces collectifs, en lien avec le nouveau cadre réglementaire du secteur. Nous conclurons enfin sur la proposition d'un schéma d'organisation de l'amont de la filière laitière biologique.

\section{Mutation du secteur laitier et spécificités des filières laitières biologiques}

Les filières agricoles doivent affronter une mutation importante due au démantèlement des outils européens de gestion des marchés. Sous pression des négociations mondiales du commerce, «l'Agenda $2000 »$ a initié la réduction des Organisations communes de marché pour les fondre dans un texte unique et remettre en cause progressivement les outils de régulation. La décennie 2000-2010 a tenté de contenir cette évolution entre orientations « régulatrices » et « libérales ». Le secteur laitier est particulièrement concerné.

\section{La fin d'un système}

Les filières laitières étaient jusqu'à récemment organisées en France autour de deux piliers : les quotas laitiers européens et la recommandation interprofessionnelle des prix du lait au sein du Centre national interprofessionnel de l'économie laitière (CNIEL), interprofession reconnue par les pouvoirs publics.

Le système des quotas laitiers organisait la gestion des volumes de façon concertée entre les pouvoirs publics et les acteurs professionnels suivant une réglementation précise. L'échelon départemental fut longtemps le lieu concret de la gestion des échanges de droits à produire en France, les laiteries ou « acheteurs » étant les acteurs de sa mise en œuvre. Cette gestion française des volumes s'inscrit, jusqu'à la fin des quotas laitiers, dans un cadre européen répartissant et limitant la production de chaque État membre. Jusqu'en 2008, le prix était négocié de façon interprofessionnelle entre la Fédération nationale des producteurs laitiers (FNPL), la Fédération nationale des coopératives laitières (FNCL) et la Fédération nationale des industries laitières (FNIL), les trois familles du CNIEL. Les éleveurs laitiers n'avaient donc pas de lien direct avec « les marchés ». Ce lien se faisait uniquement par l'intermédiaire de « leur laiterie ». Le lait, produit périssable, nécessite une collecte rapide et entraîne ainsi une dépendance des éleveurs aux laiteries. Cette dépendance est accrue par le fait que les tanks à lait dans les fermes sont souvent la propriété des laiteries et non des éleveurs. Dans les zones enclavées, le coût de la collecte limite également la concurrence entre laiteries, et bien souvent l'éleveur n'a pas la possibilité de choisir son collecteur. Le système des quotas couplé à la recommandation des prix du lait par le CNIEL permettait, plus ou moins justement, de trouver un équilibre et de compenser cette méconnaissance des marchés et cette dépendance aux laiteries. La dénonciation, par la direction générale de la concurrence, de la consommation et de la répression des fraudes (DGCCRF) en 2008, de la recommandation des prix du lait et la fin annoncée des quotas laitiers entraînent un bouleversement et imposent de trouver une nouvelle organisation.

\section{Contractualisation et Organisations de producteurs}

La loi de modernisation de juillet 2010 propose de nouveaux outils aux filières dans l'objectif d'améliorer l'adéquation entre offres et demandes sur les marchés et vise, a priori, à renforcer la concertation entre acteurs des filières. Elle encourage la contractualisation entre producteurs et acheteurs, et prévoit la possibilité de la rendre obligatoire par décret en Conseil d'État ou par extension ou homologation d'un accord interprofessionnel. Cette loi encourage aussi le regroupement des 
éleveurs, avec un objectif de rééquilibrage du rapport de force avec les laiteries qui entretenaient, jusqu'ici, une relation commerciale individualisée avec l'éleveur.

Le décret du 31 décembre 2010 rend la contractualisation obligatoire entre producteur et premier acheteur dans le secteur du lait de vache, et fixe un certain nombre de clauses devant y figurer. Les coopératives sont considérées comme ayant répondu à leur obligation de contractualisation (entre producteurs et acheteurs, l'acheteur étant la coopérative) lorsqu'elles ont remis aux coopérateurs un exemplaire des statuts ou du règlement intérieur intégrant les clauses contractuelles rendues obligatoires par la loi. Pour les éleveurs livrant des laiteries privées, c'est un contrat individuel qui est proposé. Le décret permettant aux producteurs de se regrouper et d'être reconnus en tant qu' Organisation de producteurs $(\mathrm{OP})$ pour négocier collectivement les conditions de vente du contrat ne paraît qu'un an et demi plus tard, en avril 2012. Il définit les critères de reconnaissance et le rôle de ces OP. Entre les deux décrets, le " Paquet lait», un règlement européen sur la filière laitière en vue de la suppression des quotas laitiers, est paru en mars 2012. Il aménage une dérogation au droit de la concurrence qui autorise (dans les limites fixées par le Paquet lait) les OP dites de mandat (sans transfert de propriété) à négocier volumes et prix avec les laiteries. En parallèle, la gestion des droits à produire est, pour préparer l'après-quotas, passée des départements aux régions puis aux bassins laitiers. Il y a neuf bassins laitiers répertoriés en France. Ils sont géographiquement calqués sur les neuf comités régionaux interprofessionnels du CNIEL.

Ces nouveaux outils sont en phase de démarrage. Un rapport du Conseil général de l'alimentation, de l'agriculture et des espaces ruraux (CGAAER), paru en juillet 2012, pointe des insuffisances et des améliorations possibles pour rééquilibrer les relations entre éleveurs et laiteries. Elles sont en discussion entre pouvoirs publics et instances interprofessionnelles. Quoi qu'il en soit, avec la fin de l'ancien système de régulation des filières laitières françaises, un nouvel équilibre doit être trouvé. Les producteurs ne pourront plus ne pas s'intéresser à la commercialisation de leur production ou, du moins, à leur mise en marché. Ils doivent collectivement redevenir des acteurs à part entière des filières et non seulement des fournisseurs de matières premières. Cela implique un changement de rapports sociaux entre les éleveurs et les laiteries.

\section{Spécificités des filières laitières biologiques}

L'agriculture biologique est un mode de production qui repose sur des principes fondamentaux et des exigences réglementaires, entraînant des spécificités tant en termes de production que de commercialisation. En termes de production, l'agriculture biologique répond à des enjeux de préservation et d'amélioration de la qualité des sols, de l'eau, de l'air, de la biodiversité et de notre alimentation avec des exigences réglementaires identifiées. Ces dernières, notamment l'interdiction des produits phytosanitaires de synthèse ou la limitation stricte des traitements allopathiques, impliquent des pratiques agronomiques systémiques et préventives. Ceci entraîne généralement des surcoûts et des temps de travaux importants (prise en considération des particularités pédoclimatiques et saisonnières ${ }^{2}$, implantation

2. La place importante de l'herbe dans les systèmes d'élevages laitiers biologiques associée au coût élevé des grandes cultures biologiques entraînent une saisonnalité forte des élevages laitiers bio et une sensibilité importante aux aléas climatiques. 
de prairies multi-espèces et entretien de haies $^{3}$, assolements diversifiés $\left.{ }^{4} . ..\right)$. Elle contribue ainsi à l'économie des territoires par ses performances environnementales et sociales.

Une autre spécificité tient à sa réactivité face aux « signaux » des marchés. Le coût de production en agriculture bio est plus élevé qu'en conventionnel, comme le montre l'étude de l'Institut de l'élevage réalisée par Pavie (2012). L'augmentation de la production se fait essentiellement par l'arrivée de nouveaux producteurs biologiques, et non par l'augmentation des volumes produits par les élevages déjà présents. La gestion et la rentabilité globale du système en bio ne permettent pas une croissance rapide de ces volumes par les fermes biologiques. En 2008, lorsque les laiteries ont demandé aux producteurs conventionnels d'augmenter leurs volumes, encouragés par des prix à la hausse, ceux-ci les ont fait croître en quelques mois de $15 \%$. Alors que, de 2007 à 2010 (voir encadré 1), la forte demande en produits laitiers biologiques n'a pas incité une réelle hausse, car le système de production biologique ne s'y prête pas. Ainsi, une période de conversion de deux ans n'autorise pas un ajustement immédiat entre offre et demande, mais permet d'anticiper les volumes de lait bio à venir et d'estimer les volumes qui seront produits à l'année $n+1$ voire $n+2$.

En termes de filières et de marchés, il en résulte des mécanismes particuliers

3. Une vaste étude coordonnée par l'Agence Bio entre 2010 et 2012 a permis de dresser un état des lieux de «la semence biologique en France ». Une enquête (plus de 650 éleveurs utilisateurs de semences fourragères y ont répondu) a montré que $80 \%$ des prairies sont semées en mélanges et que $46 \%$ des mélanges comprennent au moins 5 espèces différentes (Agence Bio, 2012).

4. Des surfaces de grandes cultures bio sont emblavées avec 10 cultures ( $85 \%$ ) alors qu'en conventionnel, $85 \%$ des surfaces de grandes cultures le sont avec 5 espèces (Agence Bio, 11 juin 2013). d'adaptation de l'offre et de la demande. Jusqu'à présent, le manque de considération de ces spécificités a eu pour conséquence une croissance par paliers. Une augmentation de la demande entraîne une vague de conversions. Celle-ci aboutit à l'arrivée, concentrée sur une courte période, d'un volume significatif sur les marchés. Il s'ensuit une période où l'offre quantitative de lait est supérieure à la demande, ce qui déclenche un arrêt des conversions. Pourtant, ce n'est pas la demande qui faiblit, mais le développement en soubresauts qui conduit à cette inadéquation temporaire. Si la demande peut être freinée à la suite de situations sociales difficiles, elle est tendanciellement en croissance, car les produits biologiques répondent aux nouvelles attentes des consommateurs (une meilleure prise en compte de l'environnement et du social par son ancrage territorial) et aux enjeux du $\mathrm{XXI}^{\mathrm{e}}$ de mener une transition écologique des modèles de développement. Faire peser un surplus temporaire sur les marchés est néfaste à tous les acteurs des filières biologiques. Au pire, des éleveurs peuvent arrêter la production laitière biologique à force de pression sur les prix. Dans tous les cas, il y a un risque de perte de qualités sociales et environnementales des pratiques de ces acteurs. Les pratiques environnementales sont encadrées par le cahier des charges européen, mais celui-ci ne couvre pas toutes leurs diversités. Certains producteurs sous pression peuvent aussi être tentés de suivre le strict minimum du cahier des charges, voire de jouer sur des imprécisions. Ceci fragiliserait leur système en le détachant d'une approche globale et pourrait les conduire dans des impasses. Par ailleurs, il existe des marges de progrès aux différents stades des filières en termes de démarches environnementales. Celles-ci ne peuvent être explorées et développées qu'avec un minimum de capacité d'investissement et une certaine stabilité. 


\section{Encadré 1. Illustration de la croissance par palier}

Le schéma ci-dessous de l'évolution de la collecte de lait de vache biologique montre le changement d'échelle de la fin des années 1990, début 2000. Le volume collecté a quasi quadruplé en cinq ans. Les fabrications de laits liquides biologiques conditionnés ont, quant à elles, connu une croissance à deux chiffres fin des années 1990, puis plus modeste au début du siècle (inférieure à $10 \%$, mais toujours positive). Ces fabrications utilisent près de la moitié des volumes de lait biologique. En
2001, les conversions ont été stoppées et les volumes collectés sont restés stables de 2003 à 2010. Pourtant, la demande était présente et les volumes français ont manqué dès 2005 pour répondre à celle-ci. Des importations ont pallié le manque de lait bio français. Relancer la dynamique de conversion a nécessité un certain temps et s'est réellement concrétisé en 2008 à la suite du Grenelle de l'environnement. Deux ans après, en 2010, la collecte a initié sa progression.
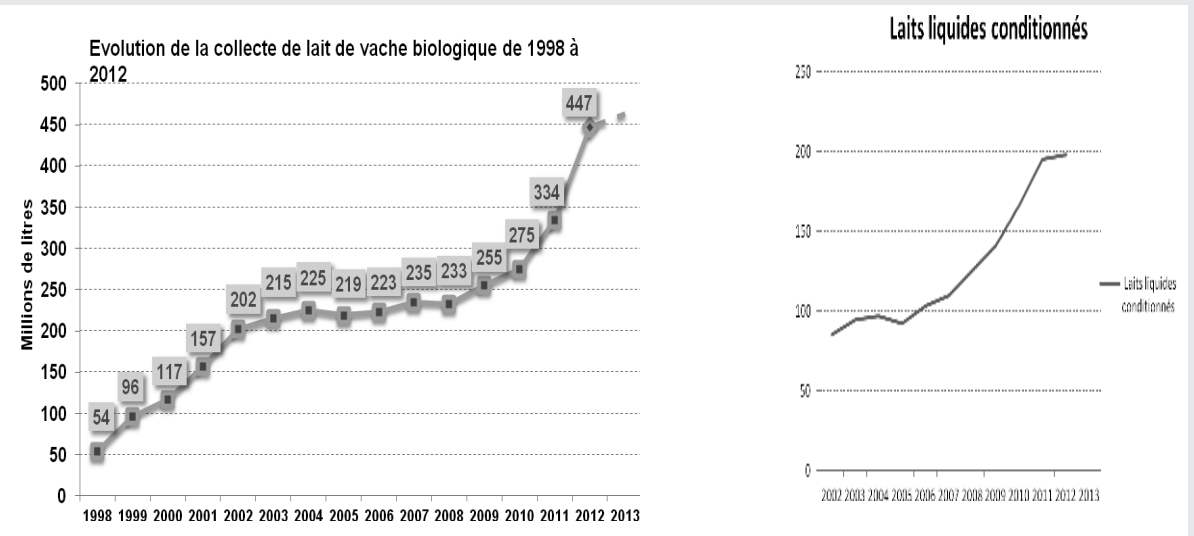

Source : Données issues des chiffres clés de l'agriculture biologique. Agence Bio (2012)

Par ailleurs, l'évolution de la demande des consommateurs en produits biologiques, et donc des marchés, ne suit pas toujours les mêmes mécanismes que celle des produits conventionnels. Les crises sanitaires des années 1990 ont entrainé une demande forte en produits bio et une vague de conversions. Celle-ci a provoqué une arrivée massive de lait biologique sur une période courte, ce qui a engendré des difficultés dans les filières biologiques, et en particulier laitière, de 2003 à 2007 (illustration de la croissance par palier). À l'inverse, en 2009, lors de la crise laitière, grâce à une demande en fait bien présente en produits laitiers biologiques, et des acteurs de l'aval encouragés par le Grenelle de l'environnement, le manque de lait bio a abouti à une augmentation de son prix. Pour les mécanismes communs comme la diminution du pouvoir d'achat, l'agriculture biologique n'a pas été affectée de la même manière ni avec la même rapidité qu'en conventionnel.

Une fois brossées les mutations du secteur agricole et laitier en particulier, les spécificités de l'agriculture biologique et sa déclinaison laitière, les deux chapitres suivants s'attacheront à montrer comment les éleveurs laitiers biologiques s'organisent pour valoriser au mieux leurs performances plurielles auprès de leurs partenaires et pour tenir compte des interactions entre les différents niveaux territoriaux dans lesquelles s'inscrivent les échanges de lait biologique. 


\section{Les premières expériences d'organisation des éleveurs laitiers bio}

Ce chapitre traite des premières initiatives d'organisation collective des éleveurs laitiers bio. Créées dans les années 1990, ces structures ont connu une phase d'expansion dans un marché peu concurrentiel, mais en création. Elles ont aussi traversé la phase de changement d'échelle de l'agriculture biologique du début des années 2000.

L'agriculture biologique a connu différentes phases de croissance. Avec la mise en place d'une réglementation officielle dans les années 1980 et 1990, elle est reconnue par les pouvoirs publics et sort de sa marginalité. En matière de commercialisation, les producteurs passant en agriculture biologique avaient peu de choix. Beaucoup vendaient en circuits courts aux consommateurs, à des magasins spécialisés ou à des entreprises de transformation, souvent $100 \%$ bio. Les années 1980-1990 ont été celles de l'émergence des Organisations économiques de producteurs bio (OEPB) et de l'implication d'acteurs agroalimentaires classiques. Les premières coopératives de grandes cultures bio sont nées dans les années 1980 (Cocebi, 1983, Biocer, 1987). Biolait est créé en 1994 sous la forme de Groupement d'intérêt économique (GIE) de collecte de lait biologique. D'autres GIE laitiers bio se mettent en place : le GIE Lait Bio du Maine, le GIE Bio Top dans les Vosges. Ils organisent la collecte de lait bio et permettent que celui-ci soit transformé et commercialisé en bio. Les agriculteurs faisant le choix de s'engager dans ce mode de production ont dû travailler à la mise en place de circuits spécifiques pour valoriser auprès des consommateurs la qualité biologique de leur production. Des agronomes, des médecins et des acteurs divers de la société civile étaient à leur côté et s'organisaient en parallèle pour acheter ces produits.
La commercialisation s'est donc inscrite dans un premier temps à une échelle locale, en lien avec les consommateurs. Puis avec les phases de croissance de la bio, certaines laiteries ont développé une collecte bio, et des éleveurs laitiers bio ont initié des GIE. Les initiatives des éleveurs pour permettre la collecte et le développement de la bio se sont faites avec deux approches différentes : l'une sur une échelle géographique proche, le territoire du bassin de vie regroupant des éleveurs d'un même département ; et l'autre à l'échelle nationale pour permettre le développement de la bio sur tous les territoires.

\section{Approche nationale Biolait, la bio partout et pour tous}

Biolait est l'expérience la plus significative de cette évolution dans les pratiques des éleveurs laitiers biologiques. Créé en 1994, Biolait rassemble, fin 2012, plus de 500 fermes, soit plus de 760 producteurs ${ }^{5}$ sur 47 départements et accompagne les producteurs en conversion. À ses origines, Biolait était un GIE, puis sa forme juridique a changé pour devenir une Société par actions simplifiées de collecte et de négoce de lait de vache biologique. Dès sa création, la finalité de Biolait était double :

- rassembler un maximum de producteurs afin de proposer une offre structurée aux transformateurs, réduire ainsi leurs charges et les livrer à la carte selon leurs besoins ;

- développer la collecte afin d'offrir la possibilité à un maximum de producteurs de se convertir en bio, quels que soient leur zone géographique et leur volume de production, en leur garantissant un prix du lait bio identique.

5. Le dénombrement des associés des fermes sociétaires, adhérentes à Biolait, explique ce nombre élevé de producteurs au regard du nombre de fermes. 
Un projet éthique, partagé

avec différents acteurs à différentes échelles territoriales

La collecte est un point central dans le développement des filières laitières. L'inscription de Biolait dans le territoire national a pour objectif de mutualiser et de réduire les coûts de collecte afin de permettre le développement de la bio partout. Mue par cette vocation, grâce à son organisation et à des accords avec d'autres laiteries, Biolait a établi des collectes dans des zones peu denses comme la Bourgogne. Pour assurer la rentabilité à terme de cette collecte, un travail d'accompagnement des producteurs voulant se convertir a été réalisé en lien avec le SEDARB (Structure de développement de l'agriculture biologique bourguignonne). Ainsi, en période favorable, Biolait développe de nouvelles collectes biologiques en zones "risquées », alors que les autres collecteurs ciblent leur accompagnement à la conversion sur des zones proches de leur usine ou renforçant leur collecte déjà en place.

Le choix de s'inscrire dans le territoire national traduit une réalité de collecte, mais aussi de filière, où les transformateurs et la distribution sont concentrés. Peser dans les négociations dans ce contexte est essentiel ; le nombre et la dimension le permettent. Pour autant, Biolait ne néglige pas les autres échelles territoriales. La transformation à la ferme et la vente directe par ses adhérents sont facilitées. Biolait livre de petites laiteries et fromageries qui ne peuvent pas développer une collecte en propre, et participe ainsi au maintien d'une diversité d'entreprises sur les territoires. Biolait a développé de nombreux partenariats avec les transformateurs aval, une quarantaine dont certains ne collectent pas de lait biologique. Ils s'inscrivent dans la durée, les contrats les encadrant sont de cinq ou trois ans.

Biolait innove dans l'établissement de partenariat avec la distribution. Cela permet d'instaurer du lien avec les consommateurs, même en filière longue. Biolait et Biocoop, réseau de magasins spécialisés en produits issus de l'agriculture biologique et proche de la production, ont développé un partenariat de filière depuis le début des années 2000. Biolait a également signé début avril 2012, à l'occasion de son assemblée générale, un partenariat avec Système U et la laiterie Saint-Denisde-l'Hôtel (département du Loiret). Ces partenariats avec la distribution spécialisée en premier lieu, puis avec Système U, sont remarquables à plusieurs égards. D'abord, ils décloisonnent les discussions entre les différents maillons de la filière : organisation de producteurs, transformateurs et distributeurs. Ensuite, ils reposent sur un projet clair multidimensionnel de développement de la bio, mais aussi d'établissement de nouveaux rapports socio-économiques ; ce projet est partagé au-delà du simple acte commercial. Économie, rapports sociaux et environnement composent différents axes du projet. Ces dimensions se traduisent par une transparence des coûts, un affichage des acteurs de la filière jusqu' au consommateur, et la participation financière des distributeurs pour encourager les conversions.

Par ailleurs, Biolait entretient depuis dix ans des relations avec des organisations européennes d'éleveurs laitiers biologiques, et a développé un partenariat avec Omsco, une coopérative bio laitière qui collecte $75 \%$ des volumes bio en Angleterre. En 2010-2011, lorsque la France manquait de lait biologique, Biolait en achetait à Omsco. Le prix du lait bio était à la hausse en France alors qu'il baissait en Angleterre, à la suite de l'effet précoce de la crise sur la consommation. Ces achats représentaient un débouché opportun pour la coopérative anglo-saxonne et permettaient à Biolait de poursuivre ses relations commerciales. 


\section{Approche à l'échelle d'un bassin de vie}

Dans les années 1990, la création de GIE de collecte par des éleveurs laitiers biologiques est liée à des questions de proximité géographique et relationnelle. Ainsi, le GIE Lait Bio du Maine, créé en 1994, collecte du lait bio et construit des partenariats avec de petites laiteries locales. Son objectif est de rester proche de ses adhérents, de s'inscrire dans un territoire, de maintenir des fermes en activité et de tisser un lien social fort entre adhérents et consommateurs. Dans cette optique, le GIE se pose la question, dans les années 2000, de la transformation du lait. Les difficultés de la filière laitière bio des années 2002 à 2006 l'incitent également à trouver un moyen de s'autonomiser, au moins en partie, des fluctuations des marchés. Le GIE se mue alors en coopérative et cherche quel produit sera à même de consolider son identité locale et de valoriser son ancrage territorial. Après plusieurs essais et recherches, c'est en puisant dans le patrimoine culturel de leur département que les éleveurs redonnent vie à un fromage local, « l'entrammes ». Ils veulent inscrire sa production et sa commercialisation au niveau local. Pour cela, ils mettent en œuvre des partenariats avec les magasins «Intermarché » locaux et avec des magasins spécialisés, réservant à ces derniers les fromages «grand cru ». En 2012, la coopérative rassemble près de 50 adhérents qui produisent plus de 10 millions de litres de lait. La fromagerie, créée par le GIE, transforme environ $10 \%$ de la production de ses adhérents. Sa capacité est du double de cette production. Quoi qu'il en soit, $80 \%$ $\mathrm{du}$ lait restera à commercialiser en vrac. Une partie l'est auprès des laiteries partenaires proches, mais sur un marché national et donc sensible à ses soubresauts. La Coop Lait Bio du Maine doit aussi prendre en compte cette échelle. C'est encore la qualité qui est privilégiée en s'investissant dans Bio Cohérence ${ }^{6}$, marque collective d'acteurs des filières biologiques. Son approche restreinte à un territoire et à un groupe de producteurs très impliqués facilite l'engagement de tous les coopérateurs dans cette démarche innovante.

Les acteurs de la Coop Lait bio du Maine portent un projet de territoire fort en termes de dynamiques sociales, culturelles et environnementales. En effet, ils privilégient la multiplication de petites structures d'exploitation plutôt que leur concentration, ce qui autorise des maintiens ou des créations d'emplois. En matière de culture, le projet coopératif a puisé dans les ressources patrimoniales locales pour faire revivre un fromage. Enfin, les bâtiments de transformation ont été construits avec des matériaux écologiques et fonctionnent entièrement avec des énergies renouvelables.

\section{Les organisations de producteurs bio de mandat}

$\mathrm{Au}$ milieu des années 2000, d'autres réflexions et démarches ont émané des producteurs bio, livrant des laiteries privées notamment. Les difficultés de valorisation connues par les filières laitières biologiques à partir de 2002/03 ont incité les éleveurs, qui ne l'avaient pas encore fait, à s'interroger sur leur rôle dans les filières de commercialisation. La Caisse de mutualisation du Grand Est (Camuge) s'est mise en place en Lorraine, des syndicats de vente ont émergé un peu plus tard en Franche-Comté. Le processus s'est accéléré avec la loi de modernisation de l'agriculture et de la pêche de 2010, et de nombreuses associations d'éleveurs bio

6. Bio Cohérence est une marque née d'une dynamique collective impliquant producteurs bio, transformateurs et distributeurs spécialisés dans les produits issus de l'agriculture biologique. Attachée à un cahier des charges strict, elle valorise des pratiques exigeantes, reflet d'une éthique et d'une démarche globale favorisant une bio de qualité, solidaire et à taille humaine. 
se sont créées, notamment dans l'Ouest de la France.

\section{Caisse de mutualisation du Grand Est}

La caisse de solidarité a été créée en juillet 2003 comme une solution d'urgence aux problèmes des producteurs. Sur une production totale régionale de 25 millions de litres de lait bio (par 124 éleveurs laitiers bio), 7 millions n'étaient pas valorisés en bio, soit $28 \%$ de la production. À la suite de la décision de deux laiteries de supprimer la prime bio, de nombreux producteurs n'ont plus eu aucune valorisation pour leur lait. Cette situation mettait en danger les producteurs concernés, mais également l'avenir d'une filière laitière biologique dans les Vosges. Ainsi, les producteurs du Groupement d'agriculture biologique de Lorraine (CGA), groupement adhérant à la FNAB, se sont penchés sur ce problème et ont proposé la création d'une caisse de solidarité (voir encadré 2).

Les principaux objectifs de la caisse de solidarité étaient de maintenir à la fois les éleveurs en bio et aussi un volume de lait certifié bio disponible, afin de répondre à la demande future. Les producteurs lorrains ont souhaité impliquer les laiteries locales dans la mise en place de la caisse, mais celles-ci ont toutes refusé. Parmi les 125 éleveurs laitiers bio de la région, 50 exploitants ont adhéré à la caisse, soit $40 \%$. C'étaient essentiellement des Vosgiens.

Le projet créé initialement pour une durée maximum de 12 mois se prolongea 3 ans sous cette forme. Il a permis de resserrer les liens entre les producteurs. Dès 2006, prévoyant l'évolution favorable du secteur laitier biologique, le groupe, à l'aide du CGA, a consolidé sa réflexion en termes de rationalisation de la collecte sur leur zone.

\section{Évolution du système vers une caisse indépendante de mutualisation \\ des primes bio}

Ayant réalisé une cartographie précise des éleveurs laitiers bio des Vosges, le CGA a mis autour de la table les différentes

\section{Encadré 2. Fonctionnement du dispositif de 2003 à 200}

Géré par le CGA Lorraine, le dispositif fournissait aux laiteries la liste des producteurs adhérents à la caisse, ainsi que les autorisations de prélèvement signées par chacun d'eux. Les laiteries prélevaient la cotisation de $3 €$ par 1000 litres directement sur le prix $\mathrm{du}$ lait des producteurs, puis les reversaient via le CGA. La caisse permettait de verser une prime aux producteurs adhérents ne percevant aucune plus-value pour leur lait bio, et ne touchant pas d'aide à la conversion ${ }^{1}$. L'aide financière était calculée par litre produit et plafonnée à 100000 litres par Unité

1. Les aides aux maintiens à l'agriculture biologique n'existaient pas et celles à la conversion étaient versées sur cinq ans. Après deux ans de conversion, le lait peut être valorisé dans les filières bio. L'idée de la caisse était de soutenir ceux qui n'avaient aucune plus value liée au mode de production biologique ni par la continuité de leurs aides à la conversion ni par le prix du lait. de travail humain (UTH). En outre, la caisse prévoyait de participer, dans la mesure des fonds disponibles, à des actions de promotion et de communication en faveur du développement de la consommation des produits laitiers bio. Les bénéficiaires de l'aide s'engageaient à retirer leur certificat bio à leur laiterie et à livrer leur lait à toute laiterie qui le valoriserait en bio après agrément de la caisse. Ainsi, cette dernière permettait de rendre disponible $\mathrm{du}$ lait bio et de regrouper les litrages non valorisés dispersés dans les laiteries. Si ces clauses n'étaient pas respectées, les producteurs étaient tenus de rembourser les 24 derniers mois de l'aide qu'ils avaient perçue. Pour devenir bénéficiaire, il fallait avoir cotisé au moins une année entière, sauf pour ceux qui étaient bénéficiaires dès la création de la caisse. Il y a eu six bénéficiaires de la caisse qui ont perçu chacun entre 0.0118 et $0.0147 €$ par litre (jusqu'à 100000 litres par UTH). 
laiteries collectant dans leur zone. Lactalis ramasse dans le nord des Vosges, Bongrain et Roussey dans le sud des Vosges. Des échanges de collecte avec du lait conventionnel sont alors discutés. Le CGA propose des collectes cohérentes pour chacune des laiteries, assurant à chacune une densité correcte de collecte et optimisant globalement les kilomètres liés à cellesci. En contrepartie, le CGA a demandé à gérer les primes bio pour les producteurs de la caisse. Le résultat des négociations a abouti à une répartition de la collecte entre les laiteries régionales et à l'engagement de chacune d'elles de reverser la prime bio des adhérents à la caisse, c'est-à-dire au CGA Lorraine. La caisse permet ainsi la mutualisation des primes entre tous les producteurs adhérents, et donc une plus value bio pour les quelques éleveurs trop excentrés pour entrer dans une collecte spécifique. En 2009, deux éleveurs étaient concernés.

Début 2008, des tensions apparaissent avec les collecteurs. Ceux-ci cherchaient à développer leur collecte de lait biologique. Le CGA Lorraine propose alors aux laiteries de participer à ses actions de développement et de conversions. Des journées par laiterie sont organisées pour présenter la bio aux producteurs conventionnels. Une participation financière pour soutenir ces actions est demandée aux laiteries liées à la caisse et cherchant du lait. Ainsi, Bongrain participe à ce travail en versant $2 €$ pour chaque 1000 litres de lait bio collecté sur la zone au cours de l'année 2008. En fin d'année, les producteurs de la caisse créent une association, la Camuge dont l'objet est la mutualisation des primes et le dialogue avec les laiteries pour des collectes cohérentes et l'incitation au développement de la bio. L'association regroupe encore essentiellement des éleveurs laitiers bio des Vosges, mais le choix de son nom laisse l'ouverture à l'adhésion d'autres producteurs. Les adhérents de la Camuge sont conscients qu'il leur faut élargir leurs bases. Des échanges réguliers ont lieu avec les éleveurs bio d'Alsace et de FrancheComté. Par contre, les discussions avec les laiteries sont plus conflictuelles, car l'enjeu autour des volumes et zones de collecte peut s'avérer être un obstacle aux négociations.

En guise de bilan, soulignons que les éleveurs vosgiens ont organisé une solidarité entre eux, le maintien en bio - grâce à la mutualisation des primes bio - de producteurs trop excentrés pour être collectés en bio. La démarche a permis d'imposer des collectes cohérentes aux laiteries présentes sur le territoire en soutenant les partenaires historiques dans l'attribution des volumes. Elle a aussi facilité l'implication financière de certaines laiteries à la dynamique de développement de la bio locale. Enfin, la caisse a participé en partenariat avec un transformateur local à l'identification d'une gamme de produits laitiers biologiques pour fournir les circuits de proximité. L'investissement avec les partenaires locaux a été fort. Par contre, les relations avec les laiteries d'envergure nationale restent difficiles, et les liens avec d'autres groupes ou éleveurs, pour renforcer la dynamique de la Camuge et avoir un poids suffisant face aux laiteries présentes à l'échelle nationale, n'ont pas abouti.

\section{Quelle configuration}

pour les OPs Bio de mandat?

La Camuge n'achète pas de lait bio et ne gère pas les quotas. Elle préfigure une OP de mandat attachée à un territoire plutôt qu'à une laiterie. Elle mutualise non l'ensemble du prix, mais la prime liée à la bio. Jusqu'à présent, elle s'est cantonnée à une échelle départementale. Élargir ses adhérents aux éleveurs des régions proches apparaît nécessaire, mais difficile. Beaucoup d'éleveurs ne veulent pas et/ou craignent de changer d'organisation. Avec la sortie du décret sur la reconnaissance d'OP dans le secteur du lait de vache, certaines laiteries font pression pour que les OP regroupent 
des éleveurs livrant une même laiterie. Les relations entre éleveurs et laiteries sont ambiguës. Les laiteries sont souvent très paternalistes envers les producteurs. Les éleveurs sont à la fois attachés et liés à leur laiterie. Ils connaissent le technicien, leur tank à lait est souvent mis à disposition par la laiterie. À grande échelle, l'évolution des rapports sociaux entre éleveurs et laiteries est progressive. En Franche-Comté, ou dans les autres régions, des regroupements s'opèrent entre éleveurs livrant la même laiterie sur un bassin donné.

Les producteurs bio livrant des laiteries privées et réunis au sein de la commission lait de la FNAB souhaitent inscrire leur structuration à plusieurs niveaux :

- une association de base regroupant les éleveurs bio livrant une même laiterie sur une zone donnée ;

- une OP de mandat « territoriale » (autant que possible) rassemblant les associations de base d'un grand bassin de production livrant différentes laiteries ;

- une fédération nationale des OP et groupes de producteurs bio, pour défendre et promouvoir les spécificités et les performances plurielles du mode de production lait bio et avoir des approches communes sur l'évolution de la production et des marchés des filières laitières biologiques.

Ce mode d'organisation collective vise à construire des partenariats équilibrés avec les laiteries aux échelles locales, mais aussi nationale, et à promouvoir le développement de l'élevage laitier bio à l'intérieur et entre bassins de production. La première OP de mandat bio inter-laiterie a été créée et reconnue en décembre 2012, c'est l'Association des producteurs laitiers bio Seine et Loire (APLB Seine\&Loire) (encadré 3).

Ces producteurs bio souhaitent partager leur projet environnemental et social avec les laiteries, mais ils sont bien conscients que certaines ont un poids important dans la collecte et la transformation biologique, bien que leur activité bio ne représente qu'une faible part de leur chiffre d'affaires, et qu'elles sont préoccupées par d'autres intérêts. La priorité est donc l'établissement de nouveaux rapports avec les laiteries et l'appropriation par les éleveurs des problématiques économiques et commerciales.

Des éleveurs laitiers bio adhérents de coopératives couplant une grande part de

\section{Encadré 3. L'APLB Seine et Loire : première OP bio de mandat}

Dans l'Ouest, de nombreuses associations de producteurs bio ont vu le jour. Elles se sont constituées par bassin et rassemblent les éleveurs livrant une même laiterie. Un travail commun et important entre éleveurs bio, la densité forte d'élevage laitier bio et la proximité avec les usines de transformation ont permis la création d'une coopération entre associations liées à différentes laiteries dans le Grand Ouest. Celle-ci regroupe sept associations de producteurs bio répartis sur la Normandie, les Pays-de-Loire et la Bretagne ( associations de base »). Plus de 200 éleveurs et environ 60 millions de litres de lait sont concernés. L'association a été reconnue OP de mandat lors de la
Commission nationale technique examinant les premiers dossiers de demande de reconnaissance dans le secteur du lait de vache. Le conseil d'administration reflète la diversité des « associations de base » la composant. Lors des rencontres à venir avec les laiteries, les représentants de 1'OP qui iront négocier avec une laiterie donnée seront des livreurs de cette laiterie. Si l'OP propose un contrat cadre, chacun pourra porter ces spécificités auprès et en lien avec son partenaire collecteur. Des éleveurs d'une même OP pourront ainsi avoir des différences de qualité et de prix payés, sur des bases discutées. Des outils d'organisation, de solidarité pourront ensuite se construire entre éleveurs. 
production conventionnelle et un peu de bio cherchent également à se réunir afin qu'une parole collective sur les spécificités de leur mode de production puisse être portée et écoutée au sein des coopératives ou groupes coopératifs. Ainsi, en 2011, les producteurs bio livrant les coopératives du groupe Eurial, se sont rassemblés en association. Environ un tiers des éleveurs bio du groupe adhère à cette association. Ils cherchent à dialoguer avec leurs coopératives et souhaitent mettre en place une section bio.

$$
*
$$$$
* *
$$

Des premières initiatives collectives des producteurs pour la mise en marché du lait biologique et des expériences plus récentes, nous tirons quelques enseignements :

- les deux approches historiques d'organisation des éleveurs laitiers bio reposent sur la volonté de porter les performances plurielles de la bio auprès de leurs partenaires économiques ;

- quelle que soit l'approche choisie, les groupes prennent en considération plusieurs échelles territoriales dans la mise en marché de leurs productions ;

- les différentes échelles d' ancrage territorial ont leur cohérence au vu du projet et de la situation actuelle des filières laitières ;

- garder une diversité des organisations facilite l'innovation et permet de tenir compte des réalités de terrain et des histoires de chacun ;

- dans l'état actuel de la production, transformation et commercialisation, l'échelle nationale, voire européenne, est fondamentale.

Un accompagnement transversal au sein de la commission lait de la FNAB permet aux éleveurs bio, quels que soient leurs horizons, de partager ces enjeux et constats. Pour conserver cette diversité, les partenariats sont construits à de multiples niveaux.

Les producteurs impliqués dans ces différentes organisations collectives se sont concertés et ont imaginé un schéma prospectif qui reste à affiner (voir annexe, figure 1). L'objectif est de fédérer le plus grand nombre d'éleveurs laitiers biologiques via les différents types de regroupements présents : associations de base et OP de mandat, coopératives bio, Biolait, groupes ou associations regroupant les éleveurs laitiers bio des coopératives ayant une activité conventionnelle et une activité biologique (mixte). Début 2013, environ $75 \%$ des éleveurs laitiers biologiques (hors vente directe) rassemblant près de $80 \%$ des volumes de collecte du lait biologique étaient adhérents à l'un de ces groupements. Environ $25 \%$ sont encore dans un rapport individuel à leur laiterie. Le nombre de producteurs et les volumes associés des adhérents de coopératives mixtes sont aussi probablement sous-évalués. Le schéma traduit le projet d'une fédération des différents groupements biologiques pour organiser la concertation.

En avril 2013, des groupements d'éleveurs laitiers biologiques ont créé Lait Bio de France. Pour l'heure, cette association fédérative réunit sept organisations économiques de producteurs bio : l'association des producteurs bio d'Eurial, Biolait, la Coop Lait Bio du Maine, l'OP Bio Seineet-Loire, le syndicat de vente Alliance, le syndicat de vente Doubs Ognon, le syndicat de vente Mullin. La FNAB en est membre de droit. Les adhérents rassemblent, à eux tous, un peu plus de 900 éleveurs (46\% des éleveurs laitiers bio hors vente directe totale) et environ 200 millions de litres de lait (48\% du volume collecté en bio en 2012).

Cette fédération a pour objectif de faire émerger de nouvelles relations avec les acteurs de l'aval de la filière et de défendre une rémunération durable des performances plurielles de la bio, dans le respect 
des règles de la concurrence. Elle aura un rôle décisif pour accompagner la professionnalisation des groupements d'éleveurs laitiers bio afin qu'ils s'inscrivent comme acteurs économiques au sein de leurs filières et territoires. Ils pourront ainsi participer efficacement à la réflexion sur la croissance de la production biologique de sorte qu'elle prenne en considération les équilibres des différents territoires.

Concrètement, elle travaillera à la création d'indicateurs sur les performances plurielles (socio-économiques et environnementales) des fermes et productions biologiques afin que chaque $\mathrm{OP}$ gérant la mise en marché de lait biologique puisse s'en saisir pour ses négociations. Elle orientera la réflexion interprofessionnelle autour des principes appropriés à la gestion de la croissance des productions laitières biologiques et proposera de nouveaux modes de relations économiques et commerciales équilibrées entre les différents acteurs des filières et des territoires.

\section{RÉFÉRENCES BIBLIOGRAPHIQUES}

Agence Bio (2012). La semence biologique en France, étude interne manuscrit (publication d'un article à venir dans le cadre de Dinabio 2013. Rey F., Sinoir N., Wohrer J., Touret C., Mazollier C., Notabili P. Semences biologiques en France : quelles pratiques, quelles attentes?)

Agence Bio (2012). L'agriculture biologique, chiffres clés. p. 164-167.

Agence Bio (2013). Quelles actions pour le développement des grandes cultures bio en France et en Allemagne pour satisfaire les besoins en alimentation humaine et animale ? Colloque Rencontres professionnelles de l'agriculture biologique, dossier manuscrit, Toulouse, juin.

Buck D., Getz C., Guthman J. (1997). From farm to table: the organic vegetable commodity chain of northern California. Sociologia ruralis, vol. $37, \mathrm{n}^{\circ} 1$, p. 3-20.

Code rural et de la pêche maritime. Partie législative, Livre VI, Titre III, Chapitre I, Section 2, Articles L631-24 à L631-26 relatifs aux contrats de vente de produits agricoles.

Commission européenne. Règlement (UE) $n^{\circ}$ 261/2012 du Parlement européen et du Conseil du 14 mars 2012 portant modification du règlement $(\mathrm{CE})$ et $n^{\circ} 1234 / 2007$ $\mathrm{du}$ Conseil en ce qui concerne les relations contractuelles dans le secteur du lait et des produits laitiers.
Décret $n^{\circ}$ 2010-1753 du 30 décembre 2010 . Pris pour l'application de l'article L. 631-24 du Code rural et de la pêche maritime dans le secteur laitier.

Décret $\mathrm{n}^{\circ} 2012-512$ du 19 avril 2012. Relatif à l'organisation économique dans le secteur du lait de vache Journal officiel du 20 avril 2012.

Conseil général de l'alimentation, de l'agriculture et des espaces ruraux (2012). Rapport $\mathrm{n}^{\circ} 12100$ sur la contractualisation dans le secteur agricole, http://agriculture.gouv. fr/IMG/pdf/Rapport-ContractualisationVdef-2_cle8169a5.pdf.

Lacombe P. (2011). Intervention au colloque de clôture de l'Assemblée générale de la Fédération nationale de l'agriculture biologique. http://fnab.org.

Le Velly R. (2009). Quel commerce équitable pour quel développement durable ? Innovations, cahiers d'économie de l'innovation, vol. $30, \mathrm{n}^{\circ} 2$, p. 99-113.

Milanesi J. (2012). Externalités et Agriculture biologique. nouvelleeconomie.blogspot.com/

Pavie J. (2012). Analyses et évolutions des coûts de production du lait en exploitations biologiques. Étude réalisée par l'Institut de l'élevage pour le CNIEL, document interne manuscrit.

Raynolds, Laura T. (2009). Mainstreaming Fair Trade Coffee: From Partnership to Traceability. World Development, vol. 37, $\mathrm{n}^{\circ}$ 6, p. 1083-1093. 


\section{ANNEXE}

Figure 1. Schéma prospectif de structuration économique des éleveurs bio

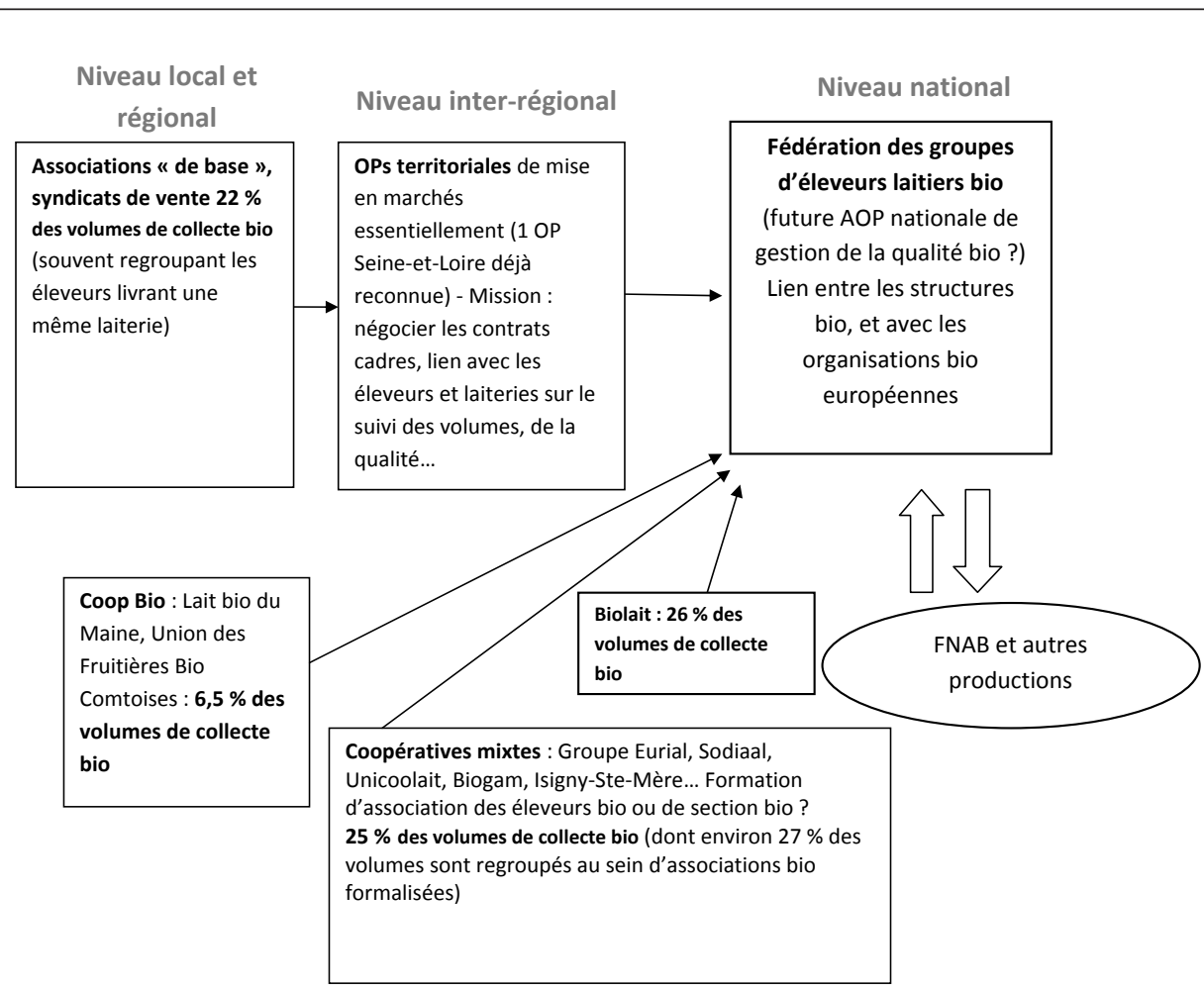

Note : Le volume de collecte des éleveurs laitiers biologiques adhérents d'un des groupes cités précédemment représente $79,5 \%$ du total de la collecte de lait biologique pour l'année 2012.

Source : Estimation au 31 décembre 2012- Flèche pleine : lien théorique d'adhésion. 